\title{
On Convex Bodies that Permit Packings of High Density
}

\author{
H. Groemer* \\ Department of Mathematics, The University of Arizona, \\ Tucson, AZ 85721, USA
}

\begin{abstract}
It is well known that an $n$-dimensional convex body permits a lattice packing of density 1 only if it is a centrally symmetric polytope of at most $2\left(2^{n}-1\right)$ facets. This article concerns itself with the associated stability problem whether a convex body that permits a packing of high density is in some sense close to such a polytope. Several inequalities that address this stability problem are proved. A corresponding theorem for coverings by two-dimensional convex bodies is also proved.
\end{abstract}

\section{Introduction}

Let $E^{n}$ denote euclidean $n$-dimensional space $(n \geq 2)$. By a convex body in $E^{n}$ we mean a compact convex subset of $E^{n}$ with interior points. If $K$ is a convex body in $E^{n}$ and $S \subset E^{n}$ the collection $\mathscr{P}(K, S)=\{K+s: s \in S\}$ is called a translative packing in $E^{n}$ if any two members of $\mathscr{P}(K, S)$ have no interior points in common. It is called a lattice packing in $E^{n}$ if there are $n$ linearly independent $b_{i} \in E^{n}$ such that $S$ consists of all points of the form $g_{1} b_{1}+\cdots+g_{n} b_{n}$ with integers $g_{i}$. We write $v(K)$ for the volume and $d(K)$ for the diameter of $K$, and let $B_{r}$ denote the (closed) ball in $E^{n}$ with radius $r$ and center at the origin $o$ of $E^{n}$. The (upper) density of a translative packing $\mathscr{P}(K, S)$ is defined by

$$
\varlimsup_{r \rightarrow \infty} \frac{1}{v\left(B_{r}\right)} \sum v(K+s)
$$

where the summation is extended over all $s \in S$ with $(K+s) \cap B_{r} \neq \varnothing$. Of course, in the case of lattice packings a much simpler definition of the density can be

\footnotetext{
* Supported by National Science Foundation Research Grants DMS 8300825 and DMS 8701893.
} 
given. We say that a convex body $K$ permits a (translative or lattice) packing of density $\delta$ if there exists a (translative or lattice) packing $\mathscr{P}(K, S)$ in $E^{n}$ of density $\delta$.

Already Minkowski [9] has shown that a convex body in $E^{n}$ that permits a lattice packing of density 1 is a centrally symmetric polytope of at most $2\left(2^{n}-1\right)$ facets. It is therefore natural to ask how much does a convex body deviate from a polytope of this kind if it permits a packing of density close to 1 ? More precisely, assuming that $K$ permits a packing of density $\delta$ we can pose the problem of finding an upper bound, possibly of the form $c(1-\delta)^{\alpha}$, on the Hausdorff distance between $K$ and such a special polytope. However, this formulation disregards a serious problem since the density of a packing is invariant under affine transformations but Hausdorff distance is not. Obviously, we can obtain rather meaningless estimates by permitting large affine distortions. To avoid such distortions we require that at least one of the two bodies whose mutual distance is under consideration be "normalized" in the following sense: a convex body $K$ in $E^{n}$ will be called normalized if $v(K)=1$ and $d(K)$ is minimal among all affine copies of $K$ of volume 1 .

It will be convenient to treat the questions of approximation by symmetric bodies and by polytopes separately (in Sections 2 and 3, respectively). A combination of both results is presented in Section 3 as a corollary. Corresponding results for coverings instead of packings appear to be much more difficult to prove. In Section 4 a covering result for the special case $n=2$ is proved. Finally, in Section 5 , some open problems are discussed.

If $K$ and $L$ are convex bodies, then $h(K, L)$ denotes always the Hausdorff distance between $K$ and $L$.

\section{Approximation by Centrally Symmetric Bodies}

If a convex body $K$ in $E^{n}$ permits a packing of density 1 the fact that $K$ is centrally symmetric is proved by applying the Brunn-Minkowski theorem to the convex body obtained from $K$ by central symmetrization, i.e., to the convex body

$$
K^{*}=\frac{1}{2}(K+(-K))
$$

Consequently, the proof of our stability result depends on a recently established stability version of the Brunn-Minkowski theorem. We formulate it here as a lemma. Its proof is given in [6, Theorem 5].

Lemma 1. Let $K$ be a convex body in $E^{n}$ and assume that for some $\varepsilon \geq 0$

$$
v\left(K^{*}\right) \leq(1+\varepsilon) v(K) \text {. }
$$

Then, the translate of $K^{*}$, say $K^{0}$, which has the same centroid as $K$ has the property that

$$
h\left(K, K^{0}\right) \leq \theta_{n} d(K) \varepsilon^{1 /(n+1)},
$$

where $\theta_{n}=4 \cdot 2^{1 /(n+1)} \cdot 3^{(n-1) / n} n^{n /(n+1)}<12 n$. 
Using this lemma we can easily prove the following result which holds not only for lattice packings but also for translative packings. $\kappa_{n}$ denotes the volume of the unit ball in $E^{n}$.

Theorem 1. Let $K$ be a convex body in $E^{n}$ that permits a translative packing of density $\delta$. Then there is a centrally symmetric convex body $K^{0}$ (which is the translate of $K^{*}$ whose center coincides with the centroid of $K$ ) such that

$$
h\left(K, K^{0}\right) \leq \theta_{n} d(K)((1-\delta) / \delta)^{1 /(n+1)},
$$

where $\theta_{n}=4 \cdot 2^{1 /(n+1)} \cdot 3^{(n-1) / n} n^{n /(n+1)}<12 n$. Furthermore, there exist an affine copy $\tilde{K}$ of $K$ and a normalized convex body $Z$ such that $Z$ is centrally symmetric with respect to the centroid of $\tilde{K}$ and

$$
h(\tilde{K}, Z) \leq \mu_{n}(1+\delta)^{1 /(n+1)},
$$

where $\mu_{n}=\left(8 / \kappa_{n}^{1 / n}\right) 4^{1 /(n+1)} \cdot 3^{(n-1) / n} n^{(3 n+1) / 2(n+1)}<12 n^{2}$.

Proof. Let $\mathscr{P}(K, S)$ be a packing of density $\delta$. It is easy to show (see p. 69 of [10]) that $\mathscr{P}\left(K^{*}, S\right)$ forms a packing of density $\left(v\left(K^{*}\right) / v(K)\right) \delta$. Since this density is at most 1 we have $v\left(K^{*}\right) \leq v(K)(1+(1-\delta) / \delta)$. Obviously, (1) is a consequence of this inequality and Lemma 1 . To prove (2) we let $\sigma$ denote a linear transformation with the property that $v\left(\sigma K^{*}\right)=1$ and that the minimal circumscribed ellipsoid of $\sigma K^{*}$ is a ball. Then $\sigma K^{*}$ is normalized and because of a theorem of John [7] (or see [8]) $\sigma K^{*}$ contains a ball of radius $d\left(\sigma K^{*}\right) / 2 \sqrt{n}$. Hence, $\left(d\left(\sigma K^{*}\right) / 2 \sqrt{n}\right)^{n} \kappa_{n} \leq v\left(\sigma K^{*}\right)=1$, and it follows that

$$
d\left(\sigma K^{*}\right) \leq 2 \sqrt{n} / \kappa_{n}^{1 / n} .
$$

If we set $\sigma K=\tilde{K}$ and make use of the linearity of $\sigma$ we have $\sigma K^{*}=(\sigma K)^{*}=\tilde{K}^{*}$. Hence, assuming that the centroid of $K$ is 0 , we obtain from (1) and (3) that

$$
h\left(\tilde{K}, \tilde{K}^{*}\right) \leq \frac{2 \sqrt{n} \theta_{n}}{\kappa_{n}^{1 / n}}\left(\frac{1-\delta}{\delta}\right)^{1 /(n+1)} .
$$

If we now define $Z$ by $Z=\tilde{K}^{*}$ and assume $\delta \geq \frac{1}{2}$, then (2) follows immediately from (4). If $\delta<\frac{1}{2}$, then, because of (3), the right-hand side of (2) is at least $\left(d\left(\sigma K^{*}\right) / 2 \sqrt{n}\right) 8 \cdot 2^{1 /(n+1)} \cdot 3^{(n-1) / n} n^{(3 n+1) / 2(n+1)}>d\left(\sigma K^{*}\right)=d\left(\tilde{K}^{*}\right)=d(\tilde{K})$, and (2) is therefore trivially true. The estimate $\mu_{n}<12 n^{2}$ is a consequence of $\kappa_{n}^{1 / n} \geq 2 / \sqrt{n}$ which is easily obtained by inscribing a cube into the unit ball of $E^{n}$.

In the case $n=2$ one can use stronger stability versions of the BrunnMinkowski theorem and a result of Behrend [1] to derive improvements of (1) and (2). 


\section{Approximation by Polytopes}

The following theorem contains our results concerning the Hausdorff distance between centrally symmetric convex bodies that permit lattice packings of high density and polytopes of the kind mentioned before.

Theorem 2. Let $K$ be a centrally symmetric convex body that permits a lattice packing of density $\delta$. Then $K$ is contained in a polytope $P$ that is symmetric with respect to the center of $K$, has at most $2\left(2^{n}-1\right)$ facets, and has the property that

$$
h(K, P) \leq \alpha_{n} d(P)(1-\delta)^{1 / n}
$$

where $\alpha_{n}=n^{1 / n} / 2$. Furthermore, there exist an affine copy $\hat{K}$ of $K$ and a normalized polytope $Q$ of at most $2\left(2^{n}-1\right)$ facets so that $Q$ is centrally symmetric with respect to the center of $\hat{K}$ and

$$
h(\hat{K}, Q) \leq \beta_{n}(1-\delta)^{1 / n}
$$

where $\beta_{n}=\left(n / \kappa_{n}\right)^{1 / n} \sqrt{n} \leq \frac{1}{2} n^{(n+1) / n}$.

Remark. The proof of this theorem will show that in the case $n=2$ it is not necessary to assume that $K$ permits a lattice packing; it suffices to assume that $K$ permits a translative packing or only a packing by congruent copies. Moreover, we may take $\beta_{2}=1$.

Our proof of Theorem 2 is based on the following lemma which is of some independent interest.

Lemma 2. If $K$ and $L$ are concentric centrally symmetric convex bodies in $E^{n}$ and $K \subset L$, then

$$
h(L, K) \leq \frac{d(L)}{2}\left(\frac{n}{v(L)}\right)^{1 / n} v(L \backslash K)^{1 / n}
$$

Proof. Let $H_{K}$ and $H_{L}$ be parallel support planes of $K$ and $L$, respectively, such that their mutual distance is $h(K, L)$ and $K$ is not between $H_{K}$ and $H_{L}$. If $E^{n}=\left\{\left(x_{1}, \ldots, x_{n}\right): x_{i}\right.$ real $\}$ we may assume that $H_{K}$ and $H_{L}$ are orthogonal to the $x_{n}$-axis of $E^{n}$ and that $o=(0, \ldots, 0)$ is the center of both $K$ and $L$. Let $H(z)$ denote the hyperplane defined by $x_{n}=z$. We set $D=L \cap H(0)$, and if $p$ is an arbitrary but fixed point of $L \cap H_{L}$ we consider the cone $C=\operatorname{conv}(D \cup\{p\})$. Let $C^{\prime}$ denote the subcone of $C$ that has also $p$ as apex but $C \cap H_{L}$ as base. Because of $C^{\prime} \subset L \backslash K$ and the symmetry of $K$ and $L$ we have

$$
2 v\left(C^{\prime}\right) \leq v(L \backslash K)
$$


Letting $v_{n-1}$ denote volume in $E^{n-1}$ we find

$$
v(L)=\int_{-\infty}^{\infty} v_{n-1}(L \cap H(z)) d z \leq d(L) \max _{z} v_{n-1}(L \cap H(z)) .
$$

If $v_{n-1}(L \cap H(z))$ attains its maximum at some value $z_{0}$ it attains it also at $-z_{0}$ and therefore (by the Brunn-Minkowski theorem) also at $z=0$. Hence,

$$
v(L) \leq d(L) v_{n-1}(D)
$$

Consequently, if we let $g$ denote the height of $C$ we obtain

$$
v(C) \geq \frac{g}{n} \frac{v(L)}{d(L)}
$$

Since the height of $C^{\prime}$ is $h(K, L)$ and $g \leq d(L) / 2$ it follows that

$$
v\left(C^{\prime}\right) \geq \frac{g}{n} \frac{v(L)}{d(L)} \frac{h(K, L)^{n}}{g^{n}} \geq \frac{2^{n-1}}{n} \frac{v(L)}{d(L)^{n}} h(K, L)^{n} .
$$

The lemma is now an immediate consequence of this inequality and (7).

Proof of Theorem 2. It has been shown by van der Corput and Davenport [2] that if a centrally symmetric convex body $K$ in $E^{n}$ permits a lattice packing of density $\delta$ then $K$ is contained in a polytope $P$ that has at most $2\left(2^{n}-1\right)$ facets, is centrally symmetric with respect to the center of $K$, and has the further property that

$$
\frac{v(K)}{v(P)} \geq \delta
$$

Hence, $v(P \backslash K) / v(P) \leq 1-\delta$, and, setting $L=P$ we can deduce from Lemma 2 that

$$
h(K, P) \leq \frac{d(P)}{2} n^{1 / n}(1-\delta)^{1 / n}
$$

which is obviously the same as (5).

Let now $\sigma$ be an affine transformation such that $\sigma P$ is normalized. Then, analogously to (3) we have

$$
d(\sigma P) \leq 2 \sqrt{n} / \kappa_{n}^{1 / n}
$$

Hence, if we set $\sigma K=\hat{K}$ and $\sigma P=Q$, then (6) is an immediate consequence of (5) and (9). 
The remark concerning the case $n=2$ follows from the fact that for packings by congruent copies of $K$ there is a centrally symmetric polygon $P$ of at most six sides that contains $K$ and satisfies (8) (see [5, p. 339]) and that according to Behrend [1] (9) can be replaced by $d(\sigma P) \leq \sqrt{2}$.

We now consider the approximation by polytopes if $K$ is not necessarily symmetric. If such a $K$ permits a packing of density $\delta$ with $\delta \geq \frac{1}{2}$, then Theorem 1 shows that there is a centrally symmetric $K^{0}$ such that

$$
h\left(K, K^{0}\right) \leq \theta_{n} 2^{1 /(n+1)} d(K)(1-\delta)^{1 /(n+1)} .
$$

Furthermore, because of Theorem 2, there is a centrally symmetric polytope $P$ of at most $2\left(2^{n}-1\right)$ facets with $K^{0} \subset P$ and

$$
h\left(K^{0}, P\right) \leq \alpha_{n} d(P)(1-\delta)^{1 / n} .
$$

If we note that $d(K) \leq d(P)$ we obtain, from these two inequalities,

$$
h(K, P) \leq\left(\theta_{n} 2^{1 /(n+1)}+\alpha_{n}\right) d(P)(1-\delta)^{1 /(n+1)} .
$$

If $\sigma$ is now an affine transformation that normalizes $P$, then this inequality (applied to $\sigma K$ and $\sigma P$ ) together with (9) yields the following result.

Corollary. Let $K$ be a convex body in $E^{n}$ that permits a lattice packing of density $\delta$. Then there is a centrally symmetric polytope of at most $2\left(2^{n}-1\right)$ facets such that (10) holds. Moreover, there exist a normalized polytope of this kind, say $T$, and an affine copy $K^{\prime}$ of $K$ such that

$$
h\left(K^{\prime}, T\right) \leq \gamma_{n}(1-\delta)^{1 /(n+1)},
$$

where $\gamma_{n}=\left(\theta_{n} 2^{1 /(n+1)}+\alpha_{n}\right) 2 \sqrt{n} / \kappa_{n}^{1 / n}<15 n^{2}$ and $\theta_{n}, \alpha_{n}$ are as in Theorems 1 and 2 , respectively.

(The condition $\delta \geq \frac{1}{2}$ has been omitted since in the case $\delta<\frac{1}{2}$ both inequalities are trivially true.)

\section{Coverings}

If $C$ is a convex body in $E^{n}$ and $S$ a discrete subset of $E^{n}$ and if the collection $\mathscr{P}(C, S)=\{C+s: s \in S\}$ has the property that $E^{n} \subset \bigcup_{s \in S}(C+s)$, then $\mathscr{P}(C, S)$ is called a translative covering of $E^{n}$. Analogously as in the case of packings the (lower) density of a covering $\mathscr{P}(C, S)$ is defined by

$$
\lim _{r \rightarrow \infty} \frac{1}{v\left(B_{r}\right)} \sum v(C+s)
$$


with the summation extending over all $s \in S$ with $C+s \subset B_{r}$. Although it is known that a convex body in $E^{n}$ that permits a translative covering of density 1 is again a centrally symmetric polytope of at most $2\left(2^{n}-1\right)$ facets it is much more difficult to prove stability theorems for coverings. This is due to the fact that for coverings of $E^{n}$ with $n>2$ there is neither an analogue of the van der Corput-Davenport theorem nor an analogue of the relationship between packings of a convex body and the corresponding body obtained by central symmetrization (or by any other symmetrization procedure, see [4]). Here we restrict ourselves to the case $n=2$ and prove a covering version of Theorem 2 . The term "hexagon" means a plane (convex) polytope of at most six sides.

Theorem 3. Let $C$ be a centrally symmetric convex body in $E^{2}$ that permits a translative covering of density $\delta$. Then $C$ contains a hexagon $T$ that is symmetric with respect to the center of $C$ and has the property that

$$
h(C, T) \leq \frac{1}{\sqrt{2}} d(C)\left(\frac{\delta-1}{\delta}\right)^{1 / 2} .
$$

Furthermore, there exists an affine transformation $\sigma$ such that the set $C^{\prime}=\sigma C$ is normalized and

$$
h\left(C^{\prime}, \sigma T\right) \leq(\delta-1)^{1 / 2} .
$$

Remark. This result could be generalized to coverings by congruent copies of $C$ provided that they do not "cross" (see [3] for the appropriate definitions).

Proof. It has been shown by L. Fejes Tóth (see the exposition [3, p. 339]) that under the assumptions of the theorem the convex set $C$ contains a hexagon $T$ that is symmetric with respect to the center of $C$ and such that $v(C) / v(T) \leq \delta$. Setting $L=C$ and $K=T$ we deduce from Lemma 2 that

$$
h(C, T) \leq \frac{d(C)}{2}\left(\frac{2}{v(C)}\right)^{1 / 2} v(C \backslash T)^{1 / 2} .
$$

Because of $v(C \backslash T) \leq v(C)(\delta-1) / \delta$ we obtain (11). To prove (12) let $\sigma$ be an affine transformation such that $\sigma C$ is normalized. Then, because of an inequality of Behrend [1], $d(\sigma C) \leq \sqrt{2}$ and applying (11) to $\sigma C$ and $\sigma T$ we find

$$
h(\sigma C, \sigma T) \leq\left(\frac{\delta-1}{\delta}\right)^{1 / 2} \leq(\delta-1)^{1 / 2} .
$$

\section{Related Problems}

We list here several additional problems that are suggested by our theorems and appear to be of interest. In view of the fact that the theorem of Minkowski 
mentioned in the Introduction is also true for nonlattice translative packings and coverings of density 1 we may ask whether there is an analogue of Theorem 1 for coverings, or an analogue of Theorem 2 for nonlattice packings and coverings? It is known (see [5]) that a convex body that permits a translative packing or covering of density 1 is a polytope with centrally symmetric facets. We may therefore ask if a convex body that permits a packing or covering of density close to 1 is close to a polytope with centrally symmetric facets? Even more significant is the problem of estimating the deviation of a convex body in $E^{n}$ from a translative tile under the assumption that the convex body permits a translative packing or covering of density close to 1 . We remark that translative tiles can be characterized as centrally symmetric polytopes with centrally symmetric facets that form "belts" consisting of four or six such facets (see [11, p. 299]) for a more detailed description of this result and references).

Since the Hausdorff distance is not affine invariant it is of some interest to investigate whether there are analogues of our theorems for the symmetric difference topology. Concerning Theorem 2 we have noted (in the proof of this theorem) that $v(K) / v(P) \geq \delta$. Hence, $v(P / K) \leq((1-\delta) / \delta) v(K)$; which shows that the distance between $K$ and $P$, measured by the symmetric difference metric, is at most $((1-\delta) / \delta) v(K)$. A similar version of Theorem 1 for the symmetric difference metric can apparently not be obtained by such a straightforward argument.

\section{References}

1. F. Behrend, Über die kleinste umbeschriebene und die grösste einbeschriebene Ellipse eines konvexen Bereichs. Math. Ann. 115 (1938), 379-411.

2. I. G. van der Corput and H. Davenport, On Minkowski's fundamental theorem in the geometry of numbers. Nederl. Akad. Wetensch. Proc. Ser. B 49 (1946), 701-707.

3. G. Fejes Tóth, New results in the theory of packing and covering. In Convexity and Its Applications, P. M. Gruber and J. M. Wills, Eds., pp. 318-359, Birkhäuser, Basel, 1983.

4. H. Groemer. Eine Bemerkung über die Überdeckung des Raumes mit unsymmetrischen konvexen Körpern. Monatsh. Math. 66 (1962), 410-416.

5. H. Groemer, Über Zerlegungen des Euklidischen Raumes. Math. Z. 79 (1962), 364-375.

6. H. Groemer, On the Brunn-Minkowski theorem. Geom. Dedicata, to appear.

7. F. John, Extremum problems with inequalities as subsidiary conditions. In Studies and Essays Presented to R. Courant, pp. 187-204, Interscience, New York, 1948.

8. K. Leichtewiss, Über die affine Exzentrizität konvexer Körper. Arch. Math. (Basel) 10 (1959), 187-199.

9. H. Minkowski, Geometrie der Zahlen, Teubner, Leipzig, Berlin, 1910. (Reprint: Chelsea, New York, 1953.)

10. C. A. Rogers, Packing and Covering, Cambridge University Press, Cambridge, 1964.

11. R. Schneider and W. Weil, Zonoids and related topics. In Convexity and Its Applications, P. M. Gruber and J. M. Wills, Eds., pp. 296-317, Birkhäuser, Basel, 1983. 